\title{
Detection of Senile Scleral Plaque after Head Trauma
}

\author{
Sarah Guigui, Tova Lifshitz, Amjad Baidousi, Ronit Yagev, Jaime Levy \\ Department of Ophthalmology, Soroka University Medical Center, Ben-Gurion University of \\ the Negev, Beer-Sheva, Israel
}

\begin{abstract}
Senile scleral plaques are late onset ocular calcifications, and are usually detected incidentally on orbital computed tomography scan following trauma. This report is of 4 elderly patients with senile scleral plaques who were admitted to the emergency room following trauma to the head. A head/orbital computed tomography scan was performed as part of the routine examination. In all 4 patients, computed tomography scan revealed a clear retina and no scleral perforation or foreign body intrusion, but bilateral radiodense areas were located anterior to the insertion of the horizontal rectus muscles. These areas were diagnosed as senile scleral plaques. Even though these ocular calcifications are benign, they have important prognostic implications, and should therefore be identified correctly and not confused with other ocular pathologies.
\end{abstract}

Key words: Calcification, physiologic, Sclera, Tomography, X-ray computed, Wounds and Injuries

Asian J Ophthalmol. 2011;12:229-30.

\section{Introduction}

Senile scleral plaque is a conglomerate of calcium salts that forms around the orbit. ${ }^{1}$ Senile scleral plaque usually affects people older than 50 years. No sex predominance has been observed. ${ }^{2}$ Clinically, senile scleral plaques appear on orbital computed tomography (CT) as focal areas of radiodensity typically located anterior to the insertions of the horizontal rectus muscles. ${ }^{3}$ This report is of 4 patients with senile scleral plaques admitted to the Emergency Department, Soroka University Medical Center, Beer-Sheva, Israel, for evaluation of head trauma.

\section{Case Reports}

Between March and May 2008, 4 patients older than 80 years (3 men and 1 woman) were admitted to the Emergency Department with head trauma. One patient had external signs of periorbital haematoma and subconjunctival haemorrhage. Slit-lamp examination revealed bilateral oval greyish-white areas anterior to the insertions of the medial and lateral rectus muscles.

As part of the trauma evaluation, a computed tomography (CT) scan of the head and/or orbit was performed for all patients. In all 4 patients, CT scan showed a clear and flat retina, with no sign of haemorrhage or oedema. The sclera was not perforated and no intrascleral foreign body was identified. However, an axial section of the orbital CT revealed oval radiodense areas anterior to the

Correspondence: Dr Jaime Levy, Department of Ophthalmology, Soroka University Medical Center, PO Box 151, Beer-Sheva 84101, Israel.

Tel: (972 8) 640 0379;

E-mail: ljaime@bgu.ac.il insertion of the horizontal muscles. The involvement was bilateral in all 4 patients.

The radiologist identified the plaques as calcifications of the sclera, namely senile scleral plaques (Figure 1). B-scan ultrasonography localised the senile scleral calcification anterior to the insertion of the horizontal rectus muscles (Figure 2).

\section{Discussion}

Even though the aetiology of senile scleral plaques has not been established, ${ }^{2}$ hypotheses include mechanical trauma of the ageing sclera, or damage secondary to degeneration of the rich vasculature of the episclera. ${ }^{4}$ Of prognostic importance is the fact that, apart from the episclera, no increased rate of calcification in other areas of the brain or orbit has been observed. ${ }^{4}$ The incidence of senile

Figure 1. Bilateral medial senile scleral plaques and left lateral plaque (arrows) in an 86-year-old woman admitted with head trauma.

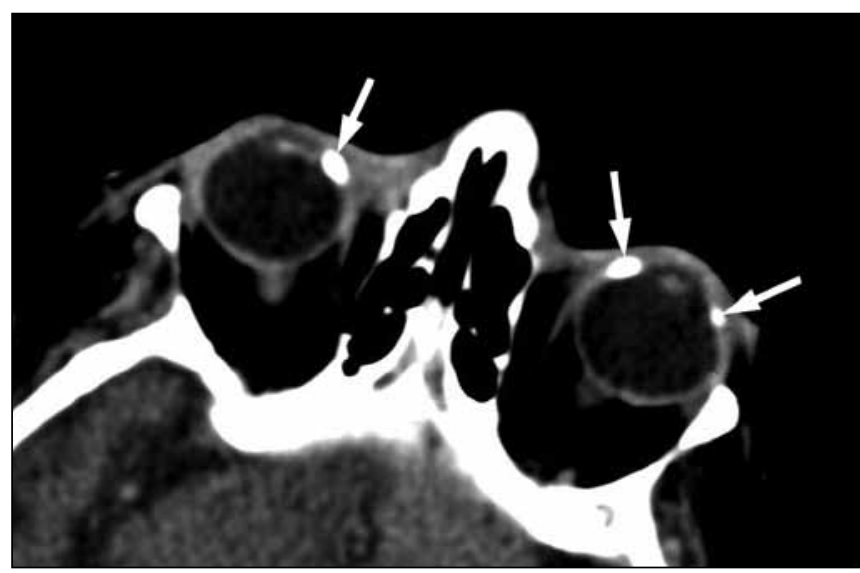


Figure 2. B-scan ultrasonography showing the senile scleral calcification (arrow) mainly anterior to the insertion of the left lateral rectus muscle in an 81-year-old man admitted with head trauma.

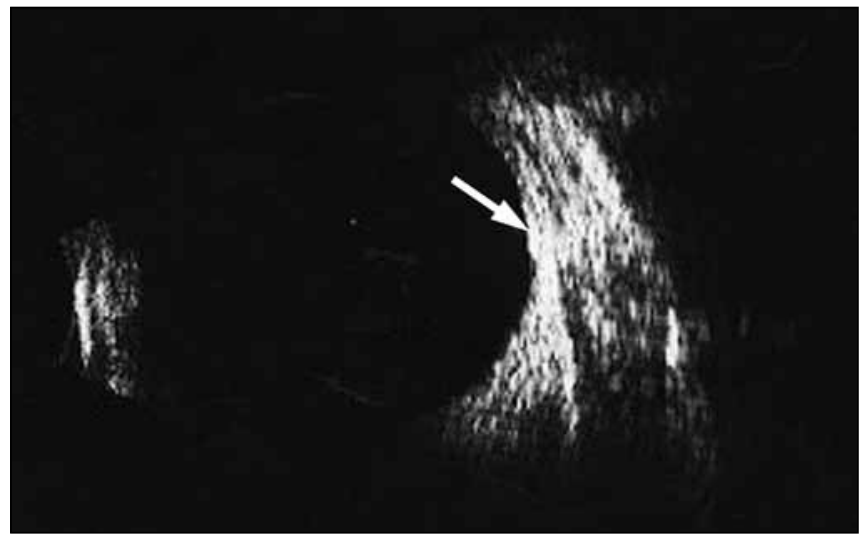

scleral plaques is unknown, but studies have shown a prevalence of $22 \%$ by CT scan in patients aged 80 years or older. ${ }^{2-5}$

Scroggs and Klintworth suggested 3 phases in the development of a senile plaque: in the first there are occasional episcleral corkscrew fibres; in the second these fibres extend progressively into the sclera; and in the third, calcification develops. ${ }^{6}$ Slit-lamp examination of eyes with early senile scleral plaques shows discrete grey discolouration of the sclera anterior to the insertion of the medial and lateral rectus muscles, that are more opaque and white when calcification occurs and in long-standing disease.

Only calcified senile scleral plaques are seen on CT, appearing as focal areas of radiodensity typically located anterior to the insertions of the horizontal rectus muscles. ${ }^{3}$ Involvement of the vertical muscles is rare. ${ }^{1}$ Ultrasound biomicroscopy does not show a 'full thickness' lesion, as could be assumed from CT imaging, but a superficial implantation above the sclera. ${ }^{4}$

The diagnosis by orbital CT scan requires an axial section showing the lens and the horizontal rectus muscles. A grey scleral discolouration on slit-lamp examination confirms the finding. ${ }^{5}$ Although for most patients, the involvement is bilateral, senile scleral plaques may occur as single lesions in up to one-third of patients. $^{1}$

Senile scleral plaques may be confused with postinflammatory scleral thinning or scleral tumour. ${ }^{6}$ In the past, these benign lesions were thought to be extraocular extensions of uveal melanomas, conjunctival malignant melanoma, necrotising scleritis, scleromalacia perforans, or postinflammatory scleral thinning. ${ }^{7}$

In trauma patients, senile scleral plaque could be mistaken for an intrascleral metallic foreign body, ${ }^{5}$ in which case the use of magnetic resonance imaging might be unnecessarily proscribed. The correct identification of senile scleral plaque has consequences for a patient's management and therefore deserves to be considered as an independent cause of orbital calcification. Characteristic findings of radiodensity, typical location anterior to horizontal muscle insertion, and bilaterality should help distinguish senile scleral plaque from other conditions.

\section{References}

1. Alorainy I. Senile scleral plaque: CT. Neuroradiology. 2000;42:145-8.

2. Moseley I. Spots before the eyes: a prevalence and clinicoradiological study of senile scleral plaques. Clin Radiol. 2000;55:198-206.

3. Goldenberg-Cohen N, Bahar I, Barash D, Naphtalaiv E, Segev Y. Sonographic features of senile scleral calcification. Ophthalmic Surg Lasers Imaging. 2007;38:115-7.

4. Cogan DG, Kuwabara T. Focal senile translucency of the sclera. Arch Ophthalmol. 1959;62:94-100.

5. Gordon RN, Slamovitz TL, Rosenbaum PS, Bello J. Calcified scleral plaques imaged on orbital computed tomography. Am J Ophthalmol. 1999;127:461-2.

6. Scroggs MW, Klintworth GK. Senile scleral plaques: a histopathologic study using energy-dispersive x-ray microanalysis. Hum Pathol. 1991;22:557-62.

7. Murthy SI, Sangwan VS. Bilateral senile scleral plaques mimicking post-inflammatory scleral ectasia. Indian J Ophthalmol. 2004;52: $59-60$ 